\title{
Journal of Clinical Orthopaedics and Trauma
}

\section{Letter to the Editor}

\section{The Emergency Department Application of a Windlass to a Bedsheet Pelvic Circumferential Compression Device}

\section{Dear Editor,}

A recently published article in your journal emphasized the importance of pelvic ring stabilization in the management of an open-book fracture. ${ }^{1}$ It has been emphasized that, because of the significant morbidity and mortality associated with this type of trauma, the early application of a pelvic circumferential compression device (PCC) can achieve fracture stabilization and reduce hemodynamic deterioration. ${ }^{2,3}$ The conclusion by Durão, et al. was that a modest maneuver with equipment such as a bedsheet can be effective in fracture stabilization. ${ }^{1}$

If one considers the bedsheet as the "strap" of a hemorrhagecontrol tourniquet, then it must also be hypothesized that the introduction of a large windlass, seen with commercial tourniquets, would improve improvised PCC efficacy. One study involving a simulated extremity hemorrhage revealed that the introduction of a windlass to a circumferential strap significantly stopped "bleeding" compared to a strap alone and the use of this adjunct has received acceptance in wilderness and military medical practice under specific circumstances. ${ }^{4}$ An earlier retrospective study questioned the efficacy of an external pelvic compression device in terms of the amount of blood transfusions and mortality. ${ }^{5}$ However, given the study's acknowledged limitations, researchers must consider developing a prospective study to evaluate the efficacy of a PCC + windlass in terms of the amount of blood transfusions and mortality.

Meanwhile, in our medical simulation of a pelvic open-book fracture, we have begun using an improvised bedsheet pelvic binder and incorporating an I.V. pole as a windlass (see attached photograph) with emphasis on accurate placement at the level of the greater trochanters. With our simulation, a bedsheet is slid under the "victim" at the level of the greater trochanters. After maximum traction of the bedsheet is achieved, an IV pole is

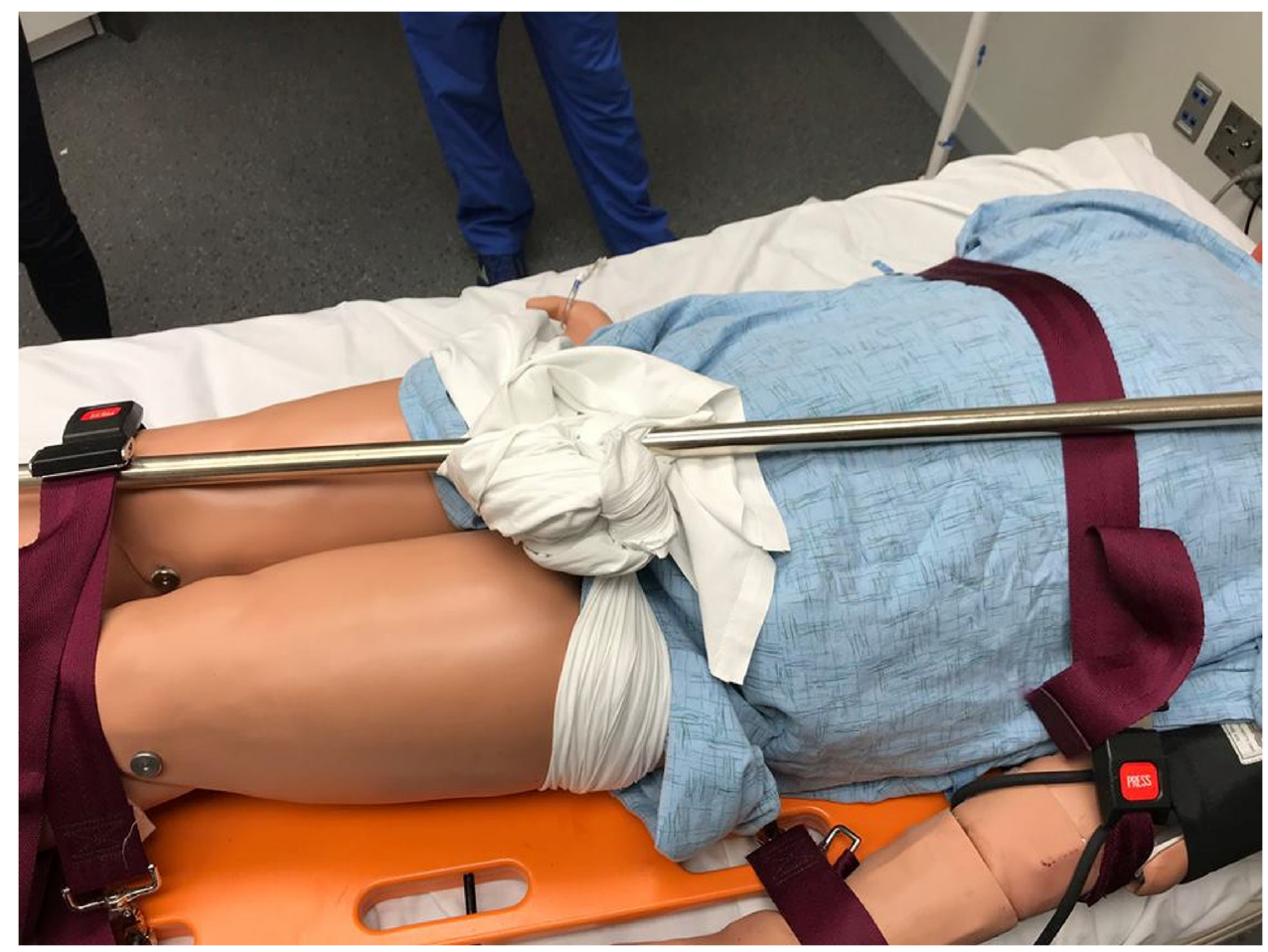

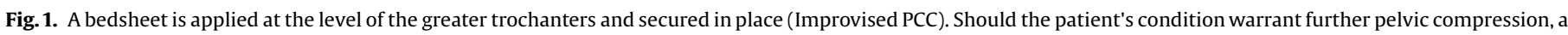
"windlass" in the form of an IV pole can be tied into the PCC and torqued to further reduce the open-book pelvic fracture. 
incorporated into the square knot to secure the sheet. The IV pole is then torqued to the maximum number of turns and secured in place with straps or another bedsheet. Binding knees and ankles coupled with a post-reduction pelvic x-ray will avoid overpressurization.

Currently, we are planning a study to evaluate its efficacy, but your readers might consider the PCC-Windlass when an improvised PCC is not having the anticipated effect on a critical patient. This might be the case in at least two specific situations: 1) The habitus of the patient (e.g. a morbidly obese patient) and 2) The physical strength of the caregivers (pre-hospital and emergency department). To avoid "windlass" injuries to both staff and patient, in-service drills should be mandated (Fig. 1).

Thank you for taking the time to consider this letter for publication in the Journal Of Clinical Orthopaedics and Trauma.

\section{Conflict of Interest}

None.

\section{References}

1. Durão C, Alves M, Barros, Pedrosa F. The importance of pelvic ring stabilization as a life-saving measure in pre-hospital - a case report commented by autopsy. $J$ Clin Orthop Trauma. 2017;8:S17-S20.
2. Halawi MJ. Pelvic ring injuries: emergency assessment and management. J Clin Orthop Trauma. 2015;6:252-258.

3. Nunn T, Cosker TD, Bose D, Pallister I. Immediate application of improvised pelvic binder as first step in extended resuscitation from life-threatening hypovolaemic shock in conscious patients with unstable pelvic injuries. Injury. 2007;38(January (1)):125-128.

4. Altamirano MP, Kragh Jr. JFJr., Aden 3rd JK3rd, Dubick MA. Role of the Windlass in Improvised Tourniquet Use on a Manikin Hemorrhage Model.J Spec Oper Med. 2015; 15:42-46.

5. Ghaemmaghami V, Sperry J, Gunst M, Friese R, Starr A, et al. Effects of early use of external pelvic compression on transfusion requirements and mortality in pelvic fractures. Amer J Surg. 2007;194:720-723.

Paul P. Rega

School of Population Health, College of Health and Human Sciences, Emergency Medicine, College of Medicine, 3000 Arlington Ave., Toledo, Ohio, 43614-2598, United StatesE-mail address: ndmsmd@aol.com (P. Rega).

Received 10 May 2018

Available online 30 June 2018 